\title{
BMJ Open Protocol for a systematic review of preference-based instruments for measuring care-related outcomes and their suitability for the palliative care setting
}

\author{
Nikki McCaffrey, ${ }^{1,2}$ Hareth Al-Janabi, ${ }^{3}$ David Currow, ${ }^{1}$ Renske Hoefman, ${ }^{4}$ \\ Julie Ratcliffe ${ }^{2}$
}

To cite: McCaffrey $\mathrm{N}$, Al-Janabi $\mathrm{H}$, Currow D, et al. Protocol for a systematic review of preference-based instruments for measuring care-related outcomes and their suitability for the palliative care setting. BMJ Open 2016;6:e012181. doi:10.1136/bmjopen-2016012181

- Prepublication history for this paper is available online. To view these files please visit the journal online (http://dx.doi.org/10.1136/ bmjopen-2016-012181).

Received 6 April 2016 Revised 16 June 2016 Accepted 20 July 2016

CrossMark

For numbered affiliations see end of article.

Correspondence to Dr Nikki McCaffrey; nicola.mccaffrey@flinders. edu.au

\begin{abstract}
Introduction: Despite informal caregivers' integral role in supporting people affected by disease or disability, economic evaluations often ignore the costs and benefits experienced by this group, especially in the palliative setting. The purpose of this systematic review is to identify preference-based instruments for measuring care-related outcomes and provide guidance on the selection of instrument in palliative care economic evaluations.
\end{abstract}

Methods and analysis: A comprehensive search of the literature will be conducted from database inception (ASSIA; CINAHL; Cochrane library including DARE, NHS EED, HTA; Econlit; Embase; PsychINFO; PubMed). Published peer-reviewed, English-language articles reporting preference-based instruments for measuring care-related outcomes in any clinical area will be included. One researcher will complete the searches and screen the results for potentially eligible studies. A randomly selected subset of $10 \%$ citations will be independently screened by two researchers. Any disagreement will be resolved by consensus among the research team. Subsequently, a supplementary search will identify studies detailing the development, valuation, validation and application of the identified instruments. The degree of suitability of the instruments for palliative economic evaluations will be assessed using criteria in the International Society for Quality of Life Research minimum standards for patient-reported outcome measures, the checklist for reporting valuation studies of multiattribute utilitybased instruments and information on the development of the instrument in the palliative setting. A narrative summary of the included studies and instruments will be provided; similarities and differences will be described and possible reasons for variations explored. Recommendations for practice on selection of instruments in palliative care economic analyses will be provided.

Ethics and dissemination: This is a planned systematic review of published literature. Therefore, ethics approval to conduct this research is not required. Findings will be presented at leading palliative
Strengths and limitations of this study

- The planned systematic review will inform guidance on the selection of informal caregiver outcome measures for economic evaluations of palliative care, promoting the inclusion of informal caregiver costs and benefits in economic analyses.

- The systematic review protocol is reported according to the recommendations of the Preferred Reporting Items for Systematic Review and Meta-Analysis Protocols (PRISMA-p) statement and is registered with the International Prospective Register of Systematic Reviews (PROSPERO).

- The total number of preference-based instruments measuring care-related outcomes may be small, limiting the study findings.

care and health economic conferences and published in a peer-reviewed journal.

Trial registration number: CRD42016034188.

\section{INTRODUCTION}

Societies increasingly, and implicitly, rely on informal caregivers' willingness to take on, maintain and repeat their caring role. ${ }^{1-3}$ Hence, informal caregivers are integral to healthcare service provision, particularly in clinical areas such as mental health, disability, dementia, cardiovascular disease, cancer, and palliative and end-of-life care. ${ }^{4-7}$ In this context, an informal caregiver is a person who provides regular, ongoing assistance to a person with progressive illness usually without receiving payment for the care they provide and could include family members, friends or neighbours. ${ }^{8}$ 
The WHO defines palliative care as, 'an approach that improves the quality of life (QOL) of patients and their families facing the problem associated with lifethreatening illness, through the prevention and relief of suffering by means of early identification and impeccable assessment and treatment of pain and other problems, physical, psychosocial and spiritual'. ${ }^{9}$ At the end of life, annual contributions by informal caregivers have been estimated at $\$ \mathrm{~A} 40$ billion ( $£ 21$ billion, $\$ \mathrm{~A} 1=£ 0.52,4$ March 2016) in Australia ${ }^{10}$ and between one-third and one-fifth of overall costs (health, social, informal care and out of pocket) in the UK and Canada, respectively. ${ }^{11}{ }^{12}$ Further, contributions by informal caregivers in the USA have been estimated at over US $\$ 500$ billion a year $\left(£ 367\right.$ billion, US $\$ 1=£ 0.71,4$ March 2016). ${ }^{13}$ Clearly, it is vital to consider the impacts on informal caregivers of different models of service provision and alternative health and social interventions when undertaking health research and formulating health policy in the palliative setting. Otherwise, cost shifting and excessive burden on family and friends could threaten the continued provision of this highly valued care. ${ }^{14}$

International, consensus-based, best practice guidelines on evaluating palliative and end-of-life care services highlight the need to include informal caregiver costs and benefits in economic evaluations. ${ }^{15}$ Broadly, economic evaluations systematically compare the relative costs and benefits of competing courses of action, providing information about how best to improve patient and family outcomes within funding constraints. Increasingly, these types of economic analyses are used to inform clinical, funding and policy decisions. ${ }^{16-19}$ Costs and benefits are considered simultaneously to evaluate how best to achieve value for money from scarce resources. ${ }^{20}$ The most commonly reported measure of benefit in economic evaluations generally is the quality-adjusted life year (QALY) ${ }^{21}$ The QALY is the sum of time spent in different quality-weighted health states, where the quality-weights represent values or preferences for alternative health states. ${ }^{22}{ }^{23}$ Often generic, preference-based measures of health-related quality of life (HrQOL) such as the EQ-5D $\mathrm{D}^{24}$ are used to generate these quality weights. ${ }^{23}$ Preference-based measures allow attributes of health to be valued differently. ${ }^{25}$ For example, an increase in mobility may be more highly valued by patients than a reduction in pain.

However, such generic measures tend to focus on health alone as the sole indicator of benefit. Other aspects of healthcare such as factors relating to the provision of services or care-specific domains, such as control, may also be valued. ${ }^{26} 27$ Consequently, economic evaluations which use generic, preference-based measures do not enable robust coverage of the impacts of interventions in complex disease areas such as palliative care where multiple, diverse domains of effect are valued. ${ }^{28}{ }^{29}$ It is vital that instruments used to measure the impact of interventions for economic evaluations are sensitive to things that matter to the patient and family.
Otherwise, measures will be unresponsive to change, leading to a suboptimal model of measurement and valuation of the true benefits of palliative strategies and misinformed decisions about where best to invest limited public funds. ${ }^{30}$

Empirical evidence indicates that conclusions relating to the cost-effectiveness of healthcare interventions can alter substantially when care-related costs and outcomes are included in economic evaluations. ${ }^{31} 32$ Despite this, impacts on informal caregivers have relatively rarely been considered in economic analyses, especially in palliative and end-of-life care. ${ }^{14} 3133$ This is due, in part, to the difficulties associated with conducting research in this context, particularly capturing and valuing caregiving effects. ${ }^{14}$

The number of economic evaluations in palliative and end-of-life care has recently started to increase. ${ }^{34-39}$ Despite this, guidance is lacking on which instruments can be used for measuring care-related outcomes in economic analyses, hindering inclusion of informal caregiver costs and benefits. Previous systematic reviews in the palliative setting have yielded many different types of instruments for capturing informal caregiver outcomes in health services research such as bereavement, burden, family functioning, mood, needs, QOL, satisfaction with service delivery and social support. ${ }^{40-42}$ However, preceding reviews did not consider the specific requirements of outcome measures in economic evaluations and may not have identified all relevant literature. Consequently, the aim of this systematic review is to identify, critically appraise and provide guidance on the selection of preference-based instruments for measuring care-related outcomes in palliative care economic evaluations.

\section{Review questions}

This systematic review aims to address three related research questions:

1. What preference-based instruments are available generally, that is, in any clinical setting, to measure care-related outcomes?

2. How have preference-based instruments measuring care-related outcomes been developed, valued, validated and applied across clinical, research and policy settings?

3. Have preference-based instruments measuring care-related outcomes been validated and valued in the palliative setting, that is, have preference weights been obtained from a representative sample of people receiving palliative care?

\section{METHODS}

A structured, three-step approach will be taken for the systematic review. First, all preference-based instruments measuring care-related outcomes in economic evaluations in any clinical setting will be identified. Based on the reviewers' knowledge of this field, the total number 
of preference-based instruments specifically developed for palliative and end-of-life care is anticipated to be small. Therefore, no restrictions regarding clinical area will be applied at this stage. Second, a supplementary search will be conducted to identify all studies reporting details on the development and application of the identified instruments, with particular attention paid to psychometric properties and valuation methods. Finally, the degree of suitability of the instruments for economic evaluations in the palliative care setting will be assessed by scoring whether the domains or dimensions were developed using input from informal caregivers of people receiving palliative care and whether items from the International Society for Quality of Life Research (ISOQOL) ${ }^{43}$ minimum standards for patient-reported outcome measures and the checklist for reporting valuation studies of multiattribute utility-based instruments (CREATE) ${ }^{44}$ have been evaluated in this population. Any protocol amendments will be documented by the lead author in the systematic review study protocol. The Flinders University Health Sciences Liaison librarian will be available to the team for the conduct of the study and specifically will provide guidance on the search strings for each database.

\section{Eligibility criteria}

Published, peer-reviewed, English-language articles reporting preference-based instruments for measuring care-related outcomes in any clinical area will be eligible for inclusion in the first step of the systematic review.

Inclusion criteria:

- Studies including informal caregivers of adult patients receiving palliative care;

- Studies reporting the development or application of a preference-based instrument for measuring carerelated outcomes;

- Primary and secondary research directly related to the topic, that is, there will be no restrictions regarding research methods;

- All geographical, national and social settings;

Exclusion criteria:

- Studies where the informal caregiver is a child or young adult or a paid caregiver, for example, healthcare professionals or paid in-home care workers;

- Informal caregivers of people receiving curative or maintenance care;

- Studies describing non-preference based care-related measures;

- Studies reporting generic (non-specific) preferencebased measures;

- Studies applying unidimensional measures;

- Non-English articles and those not peer reviewed, including editorials, comment or discussion papers, conference articles and the grey literature.

\section{Search strategy}

A comprehensive search of the literature will be conducted from database inception to the date of the search (ASSIA; CINAHL; Cochrane library including
DARE, NHS EED, HTA; Econlit; Embase; PsychINFO; and PubMed) to identify relevant published studies meeting the inclusion criteria. Keywords and $\mathrm{MeSH}$ terms, derived from previously conducted systematic reviews of informal caregiver outcome measurement tools and preference-based instruments, include following: caregiver, family, volunteer, instrument, questionnaire, outcome assessment, preference-based, utility and care-related and the search will be adapted for each database (figure 1). The reference lists of the included articles will be reviewed for additional literature unidentified by the search (pearling). Searches will be restricted to English language only. Search results and retrieved references will be deposited into Thomson Reuters EndNote V. X7.1 (2014) libraries and this software will be used to manage records throughout the review.

\section{Selection process}

First, the titles and abstracts will be reviewed by the lead investigator who has over 10 years' experience in conducting systematic reviews to assess eligibility for inclusion in the review. Full text articles will be retrieved when the abstract contains insufficient information. If necessary, corresponding authors will be contacted to ascertain whether studies meet the inclusion criteria. Second, the full text articles of potentially eligible studies will be retrieved and independently assessed from the first selection process, for inclusion in the review. A randomly selected subset of citations (10\%) will be independently screened by two other members of the research team. Agreement between the reviewers will be assessed using the Kappa statistic to determine if further duplicate reviewing is required. ${ }^{45}$ If the interrater reliability is $<0.8$, that is, strong agreement, ${ }^{45}$ an additional subset of citations $(25 \%)$ will be independently screened. If the inter-rater reliability is still $<0.8$, all remaining citations will be independently screened. Any disagreements between reviewers will be resolved by consensus among the research team.

Following identification of preference-based instruments measuring care-related outcomes, a second comprehensive search of the literature using the same databases will be conducted to identify studies reporting details on the development, valuation, measurement properties and application of the identified instruments across clinical, research and policy settings. Keywords and $\mathrm{MeSH}$ terms will include following: the name of the instrument, 'caregiver', 'valuation' and 'psychometric'. Published, peer-reviewed, English-language articles reporting preference-based, care-related instrument development, valuation, measurement properties and application will be included. The same selection process will be followed as detailed above to identify included articles.

As the total number of preference-based instruments measuring care-related outcomes is anticipated to be small, based on the reviewers' knowledge of this field, there will be no restrictions in terms of setting or study quality assessment during the screening process. 
Figure 1 EMBASE draft search strategy.

\section{Population}

1. 'caregiver'/mj OR 'family'/mj OR 'volunteer'/mj OR relative/mj OR 'care giving':ab,ti OR 'care giver':ab,ti OR caregiv*:ab,ti OR carer*:ab,ti OR volunteer*:ab,ti OR spouse*:ab,ti OR husband*:ab,ti OR wife*:ab,ti OR family:ab,ti OR informal care*:ab,ti

\section{Intervention}

2. 'health survey'/exp OR 'short survey'/exp OR 'questionnaire'/exp OR 'outcome assessment'/mj OR Questionnaire*:ab,ti OR instrument*:ab,ti OR measure*:ab,ti OR inventory:ab,ti OR inventories:ab,ti OR tool*:ab,ti OR score*:ab,ti OR indicator*:ab,ti OR scale*:ab,ti OR rating*:ab,ti OR assessment:ab,ti OR survey*:ab,ti OR instrument*:ab,ti OR measure*:ab,ti OR tool*:ab,ti

3. Preference-based

'patient preference'/exp OR 'quality adjusted life year'/exp OR 'contingent valuation'/exp OR utilit*:ab,ti OR preference*:ab,ti OR weight*:ab,ti OR "time trade off":ab,ti OR “time trade-off":ab,ti OR TTO:ab,ti OR SG:ab,ti OR "standard gamble":ab,ti OR DCE:ab,ti OR "discrete choice experiment":ab,ti OR conjoint:ab,ti OR QALY:ab,ti OR "quality adjusted life year":ab,ti OR value:ab,ti OR values:ab,ti OR valuation:ab,ti OR scor*:ab,ti OR dimension:ab,ti OR dimensions:ab,ti OR domain:ab,ti OR domains:ab,ti OR indices:ab,ti OR index:ab,ti OR attribute:ab,ti OR attributes:ab,ti

\section{Outcome}

4. 'quality of life':ab,ti OR QOL*:ab,ti OR capabilit*:ab,ti OR care-related:ab,ti OR burden*:ab,ti OR caring:ab,ti

5. (1 AND 2 AND 3 AND 4) AND limit to English

\section{Data collection}

A table will be created in Microsoft Office Excel 2010 to extract data from the included studies, summarise the evidence and inform assessment of the suitability of the preference-based instruments for measuring care-related outcomes in palliative care. This software will be used to manage data throughout the review. The form will be piloted with three of the included articles and updated accordingly. Extracted information will include following: study features (publication date, country of origin, sample size, setting (clinical, research, policy), type of study, study population, clinical area); instrument description (name of instrument, information about the instrument including domains or dimensions, number of items, description of the items and response format, administration method, ie, self-report, clinician administered, other or unknown, conceptual and measurement model, translation of the instrument, patient and investigator burden); psychometric properties (content validity, construct validity, reliability, responsiveness, floor or ceiling effects, acceptability and feasibility); and valuation (health states valued, preference elicitation technique, source for preference weights, modelling, interpretability of scores and scoring algorithm). Two reviewers will extract data independently (NM; RH). Where there is ambiguity, input will be sought from a third reviewer. Any disagreements between reviewers will be resolved by consensus among the research team.

Two reviewers will independently assess the suitability of the preference-based instruments for measuring outcomes in palliative care using the ISOQOL, minimum standards for patient-reported outcome measures (conceptual and measurement model, reliability, content validity, construct validity, responsiveness, interpretability of scores, translation of measure, patient and investigator burden), ${ }^{43}$ and the CREATE checklist (descriptive system, health states values, sampling, preference data collection, study sample, modelling, scoring algorithm). ${ }^{44}$ The ISOQOL minimum standards were chosen as these standards were developed from a systematic review of published and unpublished guidance on patient-reported outcome measures, including the COnsensus-based Standards for the selection of health Measurement Instruments (COSMIN). ${ }^{46}$ To the authors' knowledge, the CREATE checklist is the only published guidance on what key components should be reported in a valuation study. ${ }^{44}$ Information on how the contents of the instruments were developed, psychometric properties and valuation will be used to assess the suitability of the instruments for the palliative setting; instruments will be scored on whether the domains or dimensions were developed using input from informal caregivers of people receiving palliative care (yes/no) and whether each of the reporting checklist items has been evaluated for this population (if yes, then a score of one will be allocated) and a total score calculated. Any discrepancies will be identified and resolved by consensus among the research team.

\section{Data synthesis}

A narrative summary of the included studies and instruments will be provided in accordance with recent guidance from the Cochrane Collaboration. ${ }^{47}$ Four tables summarising the main features of the included studies, 
instrument descriptions, psychometric properties and valuation will be presented (see 'Data collection'). ${ }^{48}$ Using these data, similarities and differences between the instruments will be described, including their suitability for measuring care-related outcomes in palliative care and possible reasons for variations between instruments will be explored, for example, variability in study populations and method of content development. Recommendations for practice on selection of instruments for measuring care-related outcomes in palliative care economic analyses will be provided.

As the total number of preference-based instruments measuring care-related outcomes is anticipated to be small, to minimise reporting bias, the relevant development groups will be contacted to make sure no crucial information or developments have been missed.

\section{ETHICS AND DISSEMINATION}

This is a planned systematic review of published literature. Therefore, ethics approval to conduct this research is not required. The systematic review protocol is registered with the PROSPERO, registration number CRD42016034188. Findings will be presented at leading palliative care and health economic conferences and published in a peer-reviewed journal.

\author{
Author affiliations \\ ${ }^{1}$ Palliative and Supportive Services, Flinders University, Bedford Park, South \\ Australia, Australia \\ ${ }^{2}$ Flinders Health Economics Group, Flinders University, Bedford Park, South \\ Australia, Australia \\ ${ }^{3}$ Health Economics Unit, School of Health and Population Sciences, University \\ of Birmingham, Birmingham, UK \\ ${ }^{4}$ Netherlands Institute for Health Services Research, Utrecht, The Netherlands
}

Acknowledgements The coauthors warmly thank members of the Netherlands Institute for Health Services Research Scientific Committee for their constructive feedback on an earlier version of this manuscript.

Contributors NM led the conception and design of the work with input from all coauthors. NM drafted the work and the coauthors revised the protocol and manuscript critically for important intellectual content. All coauthors approved the final version to be published and are accountable for all aspects of the work. NM is the guarantor for the overall content.

Funding Funding for this study is provided by a Flinders University Early Career grant (grant number 01.574.39798) and discretionary research funds held by the respective academic departments. The funders have no role in the study design, data collection and analysis, or decision to publish.

Competing interests All members of the review team have been involved to some degree in the development, valuation, validation and application of the Carer Experience Scale (CES) and Care-related Quality of Life instrument (CarerQoL), two preference-based instruments for measuring care-related quality of life.

Provenance and peer review Not commissioned; externally peer reviewed.

Open Access This is an Open Access article distributed in accordance with the Creative Commons Attribution Non Commercial (CC BY-NC 4.0) license, which permits others to distribute, remix, adapt, build upon this work noncommercially, and license their derivative works on different terms, provided the original work is properly cited and the use is non-commercial. See: http:// creativecommons.org/licenses/by-nc/4.0/

\section{REFERENCES}

1. McCaffrey N, Currow D. Including carer effects in economic evaluations: willingness to care, a missing link? Palliat Med 2010;24: s166-7.

2. Currow DC, Burns C, Agar M, et al. Palliative caregivers who would not take on the caring role again. J Pain Symptom Manage 2011;41:661-72.

3. Johnson MJ, Allgar V, Macleod U, et al. Family caregivers who would be unwilling to provide care at the end of life again: findings from the Health Survey for England Population Survey. PLOS ONE 2016;11:e0146960.

4. Wittenberg E, Prosser LA. Disutility of illness for caregivers and families: a systematic review of the literature. Pharmacoeconomics 2013;31:489-500.

5. Currow DC. Caregivers' three-cornered hats: Their tricornes. Palliat Med 2015;29:485-6.

6. Kent EE, Rowland JH, Northouse L, et al. Caring for caregivers and patients: research and clinical priorities for informal cancer caregiving. Cancer 2016;122:1987-95

7. Torbica A, Calciolari S, Fattore G. Does informal care impact utilization of healthcare services? Evidence from a longitudinal study of stroke patients. Soc Sci Med 2015;124:29-38.

8. Al-Janabi H, McCaffrey N, Ratcliffe J. Carer preferences in economic evaluation and healthcare decision making. Patient 2013;6:235-9.

9. http://www.who.int/cancer/palliative/en/

10. Access Economics. The economic value of informal care in 2010. Sydney, NSW, Australia: Access Economics Pty Limited \& Carers, 2010.

11. Round J, Jones L, Morris S. Estimating the cost of caring for people with cancer at the end of life: a modelling study. Palliat Med 2015;29:899-907.

12. Dumont $S$, Jacobs $P$, Turcotte $V$, et al. Palliative care costs in Canada: a descriptive comparison of studies of urban and rural patients near end of life. Palliat Med 2015;29:908-17.

13. Chari AV, Engberg J, Ray KN, et al. The opportunity costs of informal elder-care in the United States: new estimates from the American Time Use Survey. Health Serv Res 2015;50:871-82.

14. McCaffrey N, Cassel JB, Coast J. Bringing the economic cost of informal caregiving into focus. Palliat Med 2015;29:866-7.

15. Evans CJ, Harding R, Higginson IJ, et al. 'Best practice' in developing and evaluating palliative and end-of-life care services: a meta-synthesis of research methods for the MORECare project. Palliat Med 2013;27:885-98.

16. Hjelmgren J, Berggren F, Andersson F. Health economic guidelines--similarities, differences and some implications. Value Health 2001;4:225-50.

17. Tarn T, Smith M. Pharmacoeconomic guidelines around the world. ISPOR connections 2004;10:5-15.

18. Drummond $\mathrm{M}$, Barbieri $\mathrm{M}$, Cook J, et al. Transferability of economic evaluations across jurisdictions: ISPOR Good Research Practices Task Force report. Value Health 2009;12:409-18.

19. Sullivan SM, Wells G, Coyle D. What guidance are economists given on how to present economic evaluations for policymakers? A Systematic Review. Value Health 2015;18:915-24.

20. Drummond M, Sculpher M, Torrance G, et al. Methods for the economic evaluation of health care programmes. 3rd edn. Oxford, UK: Oxford University Press, 2005.

21. Brazier JE, Dixon S, Ratcliffe J. The role of patient preferences in cost-effectiveness analysis: a conflict of values? Pharmacoeconomics 2009;27:705-12.

22. Neumann PJ, Goldie SJ, Weinstein MC. Preference-based measures in economic evaluation in health care. Annu Rev Public Health 2000;21:587-611.

23. Brazier J, Ratcliffe J, Tsuchiya A, et al. Measuring and valuing health benefits for economic evaluation. Oxford: Oxford University Press, 2007.

24. Herdman M, Gudex C, Lloyd A, et al. Development and preliminary testing of the new five-level version of EQ-5D (EQ-5D-5L). Qual Life Res 2011;20:1727-36.

25. Al-Janabi H, Flynn TN, Coast J. Estimation of a preference-based carer experience scale. Med Decis Mak 2011;31:458-68.

26. Ratcliffe J, Buxton M. Patient's preferences regarding the process and outcomes of high technology medicine: an application of conjoint analysis to liver transplantation. Int J Technol Assess Health Care 1999;15:340-51.

27. Al-Janabi H, Coast J, Flynn TN. What do people value when they provide unpaid care for an older person? A meta-ethnography with interview follow-up. Soc Sci Med 2008;67:111-21.

28. McCaffrey N, Agar M, Harlum J, et al. Better informing decision making with multiple outcomes cost-effectiveness analysis under uncertainty in cost-disutility space. PLOS ONE 2015;10:e0115544. 
29. McCaffrey N, Bradley S, Ratcliffe J, et al. What aspects of quality of life are important from palliative care patients' perspectives? A systematic review of qualitative research. J Pain Symptom Manage 2016:52:318-328.e5.

30. McCaffrey N, Currow DC, Eckermann S. Measuring impacts of value to patients is crucial when evaluating palliative care. $J$ Pain Symptom Manage 2009;37:e7-9.

31. Goodrich K, Kaambwa B, Al-Janabi H. The inclusion of informal care in applied economic evaluation: a review. Value Health 2012;15:975-81.

32. Krol M, Papenburg J, van Exel J. Does including informal care in economic evaluations matter? A systematic review of inclusion and impact of informal care in cost-effectiveness studies. PharmacoEconomics 2015;33:123-35.

33. Al-Janabi $\mathrm{H}$, Van Exel J, Brouwer $\mathrm{W}$, et al. Measuring health spillovers for economic evaluation: a case study in meningitis. Health Economics 2015 doi: 10.1002/hec.3259 [Epub ahead of print]

34. Farquhar MC, Prevost AT, McCrone $\mathrm{P}$, et al. Is a specialist breathlessness service more effective and cost-effective for patients with advanced cancer and their carers than standard care? Findings of a mixed-method randomised controlled trial. BMC Med 2014;12:194.

35. Serfaty M, King M, Nazareth I, et al. The clinical and cost effectiveness of cognitive behavioural therapy plus treatment as usual for the treatment of depression in advanced cancer (CanTalk): study protocol for a randomised controlled trial. Trials 2016;17:113.

36. Brandt A, Pilegaard MS, Oestergaard LG, et al. Effectiveness of the "Cancer Home-Life Intervention" on everyday activities and quality of life in people with advanced cancer living at home: a randomised controlled trial and an economic evaluation. BMC Palliat Care 2016;15:10.

37. Sahlen KG, Boman K, Brannstrom M. A cost-effectiveness study of person-centered integrated heart failure and palliative home care: based on a randomized controlled trial. Palliat Med 2016;30:296-302.

38. Agar M, Beattie E, Luckett T, et al. Pragmatic cluster randomised controlled trial of facilitated family case conferencing compared with usual care for improving end of life care and outcomes in nursing home residents with advanced dementia and their families: the IDEAL study protocol. BMC Palliat Care 2015;14:63.

39. Johnson MJ, Kanaan M, Richardson G, et al. A randomised controlled trial of three or one breathing technique training sessions for breathlessness in people with malignant lung disease. BMC Med 2015;13:213.

40. Hudson PL, Trauer T, Graham S, et al. A systematic review of instruments related to family caregivers of palliative care patients. Palliat Med 2010;24:656-68.

41. Michels CT, Boulton M, Adams A, et al. Psychometric properties of carer-reported outcome measures in palliative care: a systematic review. Palliat Med 2016;30:23-44.

42. Haines KJ, Denehy L, Skinner EH, et al. Psychosocial outcomes in informal caregivers of the critically III: a systematic review. Crit Care Med 2015;43:1112-20.

43. Reeve BB, Wyrwich KW, Wu AW, et al. ISOQOL recommends minimum standards for patient-reported outcome measures used in patient-centered outcomes and comparative effectiveness research. Qual Life Res 2013;22:1889-905.

44. Xie F, Pickard AS, Krabbe PF, et al. A checklist for reporting valuation studies of multi-attribute utility-based instruments (CREATE). Pharmacoeconomics 2015;33:867-77.

45. Orwin EG. Evaluating coding decisions. In: Cooper H, Hedges LV, eds. The handbook of research synthesis. New York: Russell Sage Foundation, 1994, pp 139-162.

46. Mokkink LB, Terwee CB, Patrick DL, et al. The COSMIN checklist for assessing the methodological quality of studies on measurement properties of health status measurement instruments: an international Delphi study. Qual Life Res 2010;19:539-49.

47. Ryan R, Cochrane Consumers and Communication Review Group Cochrane Consumers and Communication Review Group: data synthesis and analysis, June 2016. http://cccrg.cochrane.org (accessed 7 Sep 2016)

48. Green BN, Johnson CD, Adams A. Writing narrative literature reviews for peer-reviewed journals: secrets of the trade. J Chiropr Med 2006;5:101-17. 